\title{
ENERgY EFFICIENT MULTIPATH ROUTING For MOBILE AD HOC NETWORKS
}

\author{
May Cho Aye and Aye Moe Aung \\ Faculty of Information and Communication Technology, University of Technology \\ (Yatanarpon Cyber City), Pyin Oo Lwin, Myanmar
}

\begin{abstract}
Energy consumption is a significant issue in ad hoc networks since mobile nodes are battery powered. In order to prolong the lifetime of ad hoc networks, it is the most critical issue to minimize the energy consumption of nodes. In this paper, we propose an energy efficient multipath routing protocol for choosing energy efficient path. This system also considers transmission power of nodes and residual energy as energy metrics in order to maximize the network lifetime and to reduce energy consumption of mobile nodes. The objective of our proposed system is to find an optimal route based on two energy metrics while choosing a route to transfer data packets._This system is implemented by using NS-2.34. Simulation results show that the proposed routing protocol with transmission power and residual energy control mode can extend the life-span of network and can achieve higher performance when compared to traditional ad-hoc on-demand multipath distance vector (AOMDV) routing protocol.
\end{abstract}

\section{KEYWORDS}

AOMDV, energy aware routing, residual energy, transmission power control, energy efficient.

\section{INTRODUCTION}

A mobile ad hoc network (MANET) is a collection of mobile nodes with no pre-established infrastructure forming a temporary network. Each device in a MANET is free to move independently in any direction, and will therefore change its links to other devices frequently. Because of the limited transmitter range of the nodes, multiple hops may be needed to reach other nodes. Due to the mobility of the nodes, the structure of the network changes dynamically [1]. In MANET, each node participates in routing by forwarding data for other nodes, and so the determination of which nodes forward data is made dynamically based on the network connectivity. Mobile Ad Hoc networks find its application in many areas and are useful for many cases.

Routing protocols in MANETs are classified under two major fields of protocols: Proactive or table-driven and Reactive or on-demand. Some of reactive or on-demand protocols are Dynamic Source Routing (DSR), Ad-hoc On-demand Distance Vector Routing (AODV) and Ad-hoc Ondemand Multipath Distance Vector Routing (AOMDV). These protocols employ a minimum-hop metric for choosing a route and do not consider energy. DSR is a simple and on-demand routing protocol for MANET. DSR uses source routes to control the forwarding of packets through the network [2]. 
AODV [3] is an on-demand routing protocol which is essentially a combination of DSR and DSDV. In AODV, a route is established only when it is required by a source node for transmitting data packets. AOMDV is an extension to the AODV protocol; it belongs to on demand and reactive routing protocol of ad-hoc wireless networks. The main goal is to compute multiple loopfree and link-disjoint paths between source and destination pair [4].

The efficient node-energy utilization in mobile ad-hoc networks is an essential role. Death of node due to energy exhausted in ad hoc network leads to the network partition and causes communication failure in the network. Since energy is limited in wireless mobile ad hoc networks, designing energy aware routing protocols has become a main issue. The aim of these protocols is to reduce the energy consumption of the mobile nodes in the network in order to maximize the lifetime of the network. So, based on a reactive and multipath routing, we propose a new routing protocol and also consider transmission power of nodes and residual energy as energy metrics in order to maximize the network lifetime and to reduce energy consumption of mobile nodes.

The remainder of this paper is organized as follows: In section 2, we present the related works relevant to our paper. Section 3 describes a detailed description of the proposed system. In section 4 , we discuss the simulation analysis and results of the proposed system through simulation experiments and the section 5 provides the conclusion.

\section{RELATED WORKS}

There are several researches that have been done for energy efficient routing protocols in wireless mobile ad hoc network. In this section, we discuss some related works relevant to our paper.

In [5], the authors developed a new routing algorithm which named as ECNC_AODV (Energy Constraint Node Cache) based routing protocol which is derived from the AODV protocol and this algorithm is based on the current energy status of each node and the cached node.

The authors in [6] proposed the algorithm which combines two of the energy metrics and integrates these metrics into AODV in an efficient way so that the Ad hoc network has a greater life time and the energy consumption across the nodes is reduced.

In [7], the authors suggested an energy efficient multipath routing protocol for mobile ad hoc networks, called MMRE-AOMDV, which extends the standard AOMDV routing protocol. The main idea of the protocol is to find the minimal nodal residual energy of each route in the route selection process and arrange multi-route by descending nodal residual energy. Once a new route with greater nodal residual energy is emerging, it is reselected to forward rest of the data packets. It can balance individual node's energy consumption and hence prolong the entire network's lifetime.

The authors [8] proposed an optimized energy aware routing called OEAR which takes into account energy of the node and the number of packets buffered in the node while selecting the route. The proposed OEAR finds the most stable path among the existing paths from source to destination using on-demand routing. 


\section{Proposed System}

In the traditional AOMDV, it builds multiple paths using RREQs. It does not take into account the energy for choosing the paths. Here the proposed protocol not only considers residual energy but also transmission power of nodes in paths selection to maximize the lifetime of networks. The proposed system consists of three stages:

1. Control transmission power

2. Calculate residual energy

3. System operation

\subsection{Transmission Power Control}

When a node receives a packet from a neighbor, the channel attenuation is computed as the difference of the transmitted power Power $_{t x m a x}$ and the received power Power $_{r x}$. The ideal transmission power can be calculated as follows:

$$
\text { Power }_{t x}=\text { Power }_{t x m a x}-\text { Power }_{r x}+S_{r}+\text { Sec }_{t h}
$$

where $S_{r}$ is the minimal power level required for correct packet reception and $S e c_{t h}$ is the power included to overcome the problem of unstable links due to channel fluctuations [9].

In order to find the optimal path, the value $P$ can be defined as follows:

$$
P=\max _{j} \min _{i}\left(R E / \text { Power }_{t x}\right)
$$

The optimum route is determined by using the value of $P$ described above. Among all feasible paths, we choose the path with the maximum value $P$ as the optimal route for transmitting data packets. Here $R E$ is the residual energy on the route and Power $_{t x}$ is the transmission power.

\subsection{Residual Energy Calculation}

The residual energy is the remaining energy at every node which is the energy left after the packet transmission. The residual energy $R E$ can be calculated by using the following formula

$$
R E=E_{I}-E_{C}(t)
$$

where $E_{I}$ is the initial energy of a node and $E_{C}(t)$ is energy consumed by a node after time $t$. Total energy consumption of all nodes is defined as the following equation

$$
T E_{C}=N * \text { Initial Energy }-R E
$$

Here $N$ is denoted as the number of nodes used in the network.

\subsection{Operation of Proposed AOMDV}

We have proposed energy aware on-demand routing protocol for choosing energy efficient path. The goal of our proposed AOMDV is to find the optimal route which can reduce the energy consumption of mobile nodes and increase the lifetime of the network. This system discovers the 
feasible routes based on residual energy and transmission power of nodes as energy metric. This new schemes has three phases.

\subsubsection{Route Discovery}

Our proposed AOMDV protocol performs a route discovery process similar to the original AOMDV protocol. When a source node wants to send a packet to destination for which it does not already have a route, it forward a route request (RREQ) packet to all the neighbours across the network. Two additional fields called transmission power Power ${ }_{\mathrm{tx}}$ and residual energy of node $\mathrm{RE}$ are added in the RREQ header information.

The extended Route Request packet of proposed AOMDV is shown in Figure 1.

\begin{tabular}{|l|l|l|l|l|l|l|}
\hline SA & DA & Seq No & $\begin{array}{l}\text { Hop } \\
\text { Count }\end{array}$ & Timeout & Power $_{\mathrm{tx}}$ & RE \\
\hline
\end{tabular}

Figure 1. Extended route request message format of proposed AOMDV

In route discovery phase, the source node initiates the extended RREQ message to the destination node. When the destination node receives the Route Request (RREQ) packet, it will produce the Route Reply (RREP) packet and send back to the source node. The RREQ packet will be received by the intermediate nodes within the range of wireless transmission. If these nodes are not destination and do not receive the RREQ with the same packet ID, they will forward the RREQ. In this case, this proposed system applies an energy threshold functions in route discovery, in order to filter out the nodes with lower residual energy and to reduce the broadcast operations in route discovery. If the calculated energy value is greater than the threshold value of energy, the RREQ message forwards to the next neighbour node, otherwise it will be discarded. The transmission power is computed at every node in the network. When the RREQ message arrive at next node, the transmission power and residual energy is updated into the route list entries. The structure of routing table entries for AOMDV and proposed AOMDV are given in Table 1.

Table 1. Routing table entries for traditional AOMDV and proposed AOMDV

\begin{tabular}{|l|l|}
\hline \multicolumn{1}{|c|}{ Traditional AOMDV } & Proposed AOMDV \\
\hline Destination Address & Destination Address \\
\hline Sequence Number & Sequence Number \\
\hline Advertised-hop count & Advertised-hop count \\
\hline $\begin{array}{l}\text { Route List } \\
\text { (next hop1, hop count1), } \\
\text { (next hop2, hop } \\
\text { count2),.... }\}\end{array}$ & $\begin{array}{l}\text { Route List } \\
\text { (next hop1, hop count1, Power } 1 \text { 1, } \\
\text { (next hop2, hop count2, Power }\end{array} 2$, \\
& RE2),.... $\}$ \\
\hline Expiration time out & Expiration time out \\
\hline
\end{tabular}




\subsubsection{Route Selection}

In route selection phase, when the source node receives the RREPs packets from the neighbour nodes, it starts a timer and collects the RREPs during the period. Then the source node begins to calculate the values $\mathrm{P}$ based on the corresponding records in RREPs according to Eq. 2 and choose the path with the maximum value $\mathrm{P}$ as the optimal route. Finally, data packets are sent through this path with the transmission power recorded in RREP.

\subsubsection{Route Maintenance}

When a node finds a failure of route, it will send a route error (RERR) packet to the previous node to indicate the route breakage. The intermediate node which receives this RERR message informs to the source node. Then the source node will remove the corresponding item from the routing table and switch to alternate path.

\section{Performance Evaluation}

\subsection{Simulation Model and Parameters}

The system is implemented using NS-2.34 [10] to simulate our proposed algorithm. In our simulation, the channel capacity of mobile hosts is set to the same value: 2 Mbps. The testing is done in a simulation environment which is $1000 \mathrm{~m} \times 1000 \mathrm{~m}$ in area and the simulation time is 200 seconds. The speed is set as $5 \mathrm{~m} / \mathrm{s}$. The simulated traffic is Constant Bit Rate (CBR).

The simulation settings and parameters are summarized in Table 2.

Table 2. Simulation parameters

\begin{tabular}{|l|l|}
\hline Simulator & NS-2.34 \\
\hline Routing Protocols & $\begin{array}{l}\text { Traditional AOMDV } \\
\text { Proposed AOMDV }\end{array}$ \\
\hline Simulation Time (Sec) & $200 \mathrm{sec}$ \\
\hline Simulation Area & $1000 \mathrm{~m}^{*} 1000 \mathrm{~m}$ \\
\hline Number of Nodes & $25,50,100,150,200,250$ \\
\hline Transmission Range (m) & $250 \mathrm{~m}$ \\
\hline Mobility Model & Random way point \\
\hline Maximum Speed & $5 \mathrm{~m} / \mathrm{sec}$ \\
\hline Connection Rate & 5 packets/sec \\
\hline Data Packet Size & 64 bytes \\
\hline Traffic Source & CBR \\
\hline Initial Energy & 100 Joules \\
\hline
\end{tabular}




\subsection{Performance Metrics}

The performance of the proposed AOMDV protocol is compared with that of traditional AOMDV protocol according to the following metrics.

\subsubsection{Average Energy Consumption}

It is the average energy consumed by all nodes in the network.

\subsubsection{End to End Delay}

The end to end delay is the average time interval between the generation of a packet at a source node and the successfully delivery of the packet at the destination node.

\subsubsection{Throughput}

The throughput is the ratio of the data packets received at the destination to the data packets sent out from the sources.

\subsection{Performance Results}

We evaluate the performance of the two protocols in terms of average energy consumption, end to end delay and throughput as the performance metrics. We compare the proposed protocol based on transmission power of nodes and residual energy with traditional AOMDV.

Fig. 2 shows that the proposed AOMDV reduces the total energy consumption than conventional AOMDV even the number of nodes are varied.

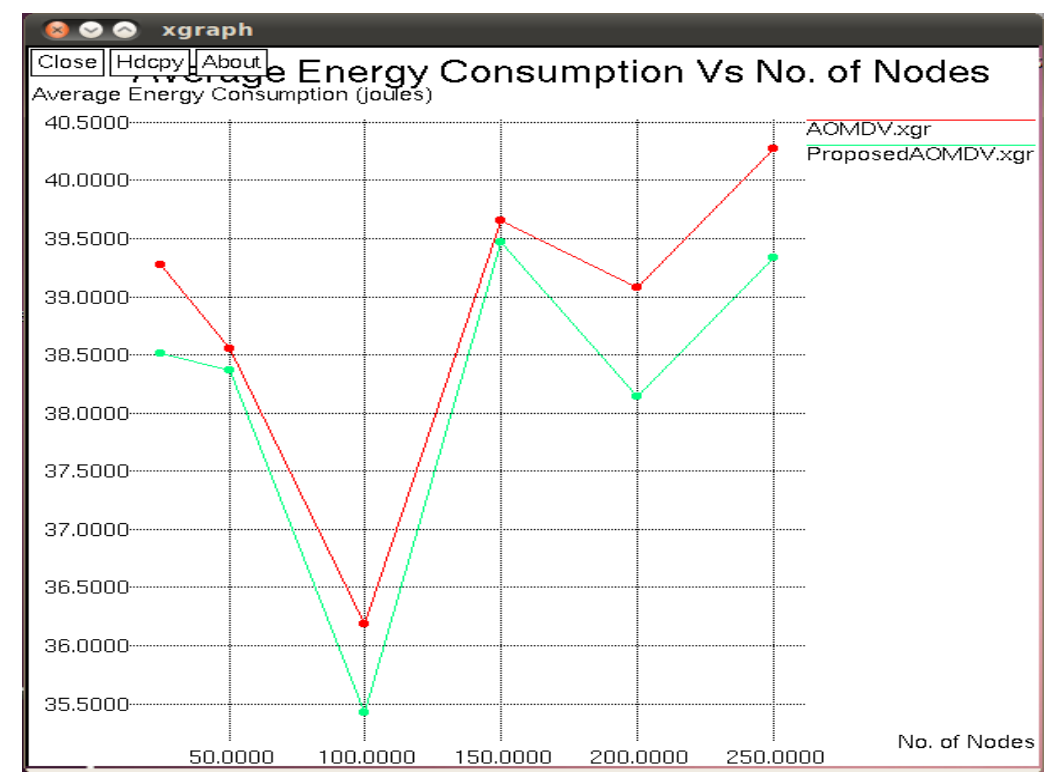

Fig. 2. Average Energy Consumption Comparison

In Fig.3, the proposed AOMDV has the lower average end-to-end delay compared to AOMDV with different number of nodes. It outperforms energy efficient communication. 


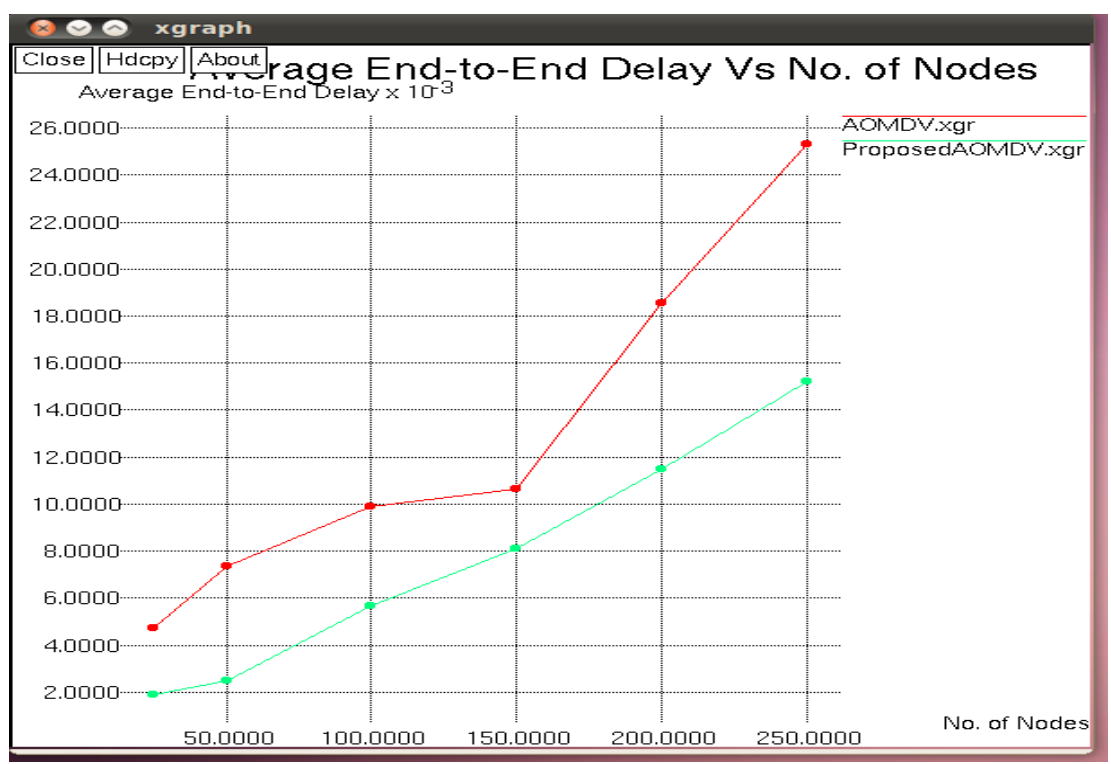

Fig. 3. Average End-to-End Delay Comparison

Fig.4 shows that the proposed AOMDV is better than original AOMDV based on throughput.

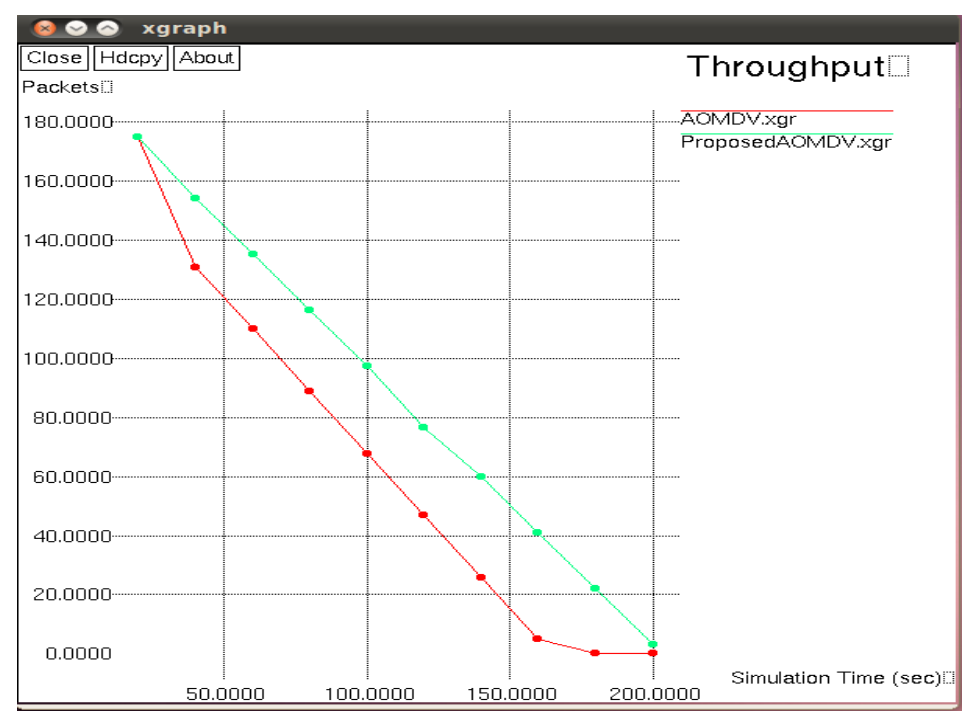

Fig. 4. Throughput Comparison

\section{Conclusions}

In this paper, we propose an energy efficient multipath routing protocol for choosing energy efficient path. The proposed algorithm considers transmission power of nodes and residual energy to extend the network lifetime and reduce the energy consumption of mobile nodes. This system is provided to reduce energy consumption and end to end delay to improve the network lifetime and throughput. 


\section{ACKNOWLEDGEMENTS}

I would especially convey her thanks and credit to supervisor, Dr. Aye Moe Aung, for her timely guidance, support and her patient participation throughout my thesis work.

\section{REFERENCES}

[1] Anne Aaron, and JieWeng, "Performance Comparison of Ad-hoc Routing Protocols for Networks with Node Energy Constraints," International Journal of engineering and technology, EE 360 Class Project Spring 2000-2001.

[2] D. B. Johnson and D. A. Maltz, "Dynamic Source Routing in Ad hoc Wireless Networks," Mobile Computing, vol.353, pp. 153-181, Kulwer Academic Publishers, 1996.

[3] C. E. Perkins, and E. M. Royer, "Ad-hoc On-Demand Distance Vector Routing," Proc. of 2nd IEEE Workshop on Mobile Computing Systems and Applications, New Orleans, LA, pp. 90-100, 1999.

[4] Mahesh K. Marina, and Samir R. Das, "Ad hoc on-demand multipath distance vector routing," Wireless Communications and Mobile Computing, Vol 6, No. 7, pp. 969-988, November 2006.

[5] Dhiraj Nitnaware, and Ajay Verma, "Energy Constraint Node Cache Based Routing Protocol for Adhoc Network," International Journal of Wireless \& Mobile Networks (IJWMN), Vol 2, No. 1, February 2010.

[6] Annapurna P Patil, Dr K Rajani kanth BatheySharanya, M P Dinesh Kumar and Malavika J, "Design of an Energy Efficient Routing Protocol for MANETs based on AODV," IJCSI International Journal of Computer Science Issues, Vol 8, Issue 4, No. 1, July 2011, ISSN (Online): 1694-0814.

[7] Yumei Liu, Lili Guo, Huizhu Ma and Tao Jiang, "Energy Efficient on-demand Multipath Routing Protocol for Multi-hop Ad Hoc Networks," 978-1-4244-2204-3/08/ @2008 IEEE.

[8] Seema Verma, Rekha Agarwal, and Pinki Nayak, "An Optimized Energy Aware Routing (OEAR) Scheme for Mobile Ad Hoc Networks using Variable Transmission Range," International Journal of Computer Applications (0975-8887), Vol 45, No. 12, May 2012.

[9] P. Bergamo, "Distributed Power Control for Energy Efficient Routing in Ad Hoc Networks," Wireless Networks, pp. 29-42, 2004.

[10] The VINT Project, "Network simulator_ns2," http://www.isi.edu/nsnam/ns/.

\section{Authors}

Ms. May Cho Aye is an Assistant Lecturer at West Yangon Technological University (WYTU). She has received her B.E degree in Information Technology from Technological University (Taungoo), Myanmar in 2006 and M.E degree in Information Technology from West Yangon Technological University (WYTU), Myanmar in 2010. Currently, she is pursuing Ph.D degree in Information Technology from University of Technology (Yatanarpon Cyber City), Pyin Oo Lwin, Myanmar. Her research interests are Wireless Communications, Mobile Ad Hoc Networks and Wireless Sensor Networks.

Dr. Aye Moe Aung is with the Faculty of Information and Communication Technology, University of Technology (Yatanarpon Cyber City), Pyin Oo Lwin, Myanmar. 